\title{
Téoros
}

Revue de recherche en tourisme

\section{Le programme « Rues Principales » et le développement du tourisme culturel}

\section{Claude Moulin}

Volume 7, numéro 1, mars 1988

Cultures régionales et tourisme

URI : https://id.erudit.org/iderudit/1080431ar

DOI : https://doi.org/10.7202/1080431ar

Aller au sommaire du numéro

Éditeur(s)

Université du Québec à Montréal

ISSN

0712-8657 (imprimé)

1923-2705 (numérique)

Découvrir la revue

Citer cet article

Moulin, C. (1988). Le programme « Rues Principales » et le développement du tourisme culturel. Téoros, 7(1), 30-31. https://doi.org/10.7202/1080431ar d'utilisation que vous pouvez consulter en ligne.

https://apropos.erudit.org/fr/usagers/politique-dutilisation/ 
La Fondation canadienne pour la protection du patrimoine est l'initiatrice d'un programme de revitalisation des centres-villes nomme "Rues Principales", Ces quartiers revitalisés deviennent ensuite des attractions touristiques et ce tourisme, graduellement, rejoint les lignes directrices de ce qu'on appelle le tourisme culturel ou patrimonial.

Cet article traite des rapports entre les exigences de la revalorisation des centres-villes et celles du developpement touristique. Ces questions deviendront d'autant plus pertinentes que 70 projets de Rues Principales se réaliseront au Canada d'ici 1991.

\section{Naissance et évolution du projet Rues Principales}

La construction des centres d'achats à la periphérie des villes a entraîné une désertification des centres-villes. On retrouve ce phénomène à la grandeur du continent nordaméricain. Quelques projets au début des années 1970, notamment dans l'ouest canadien (Alberta), avaient pour objectif de redonner une certaine esthétique au centreville. Mais il s'agissait d'opérations ponctuelles, plutôt cosmétiques, et qui n'avaient pas attaqué dans son essence toute la complexité des liens sociaux, culturels et patrimoniaux ni les problèmes de fond, base de la perte de la force d'attraction du centre-ville.

La Fondation canadienne pour la protection du patrimoine choisit Perth en Ontario en 1979 pour initier un projet-pilote de revitalisation de la Rue Principale. Elle y installa un responsable avec un burea! sur la rue principale qui s'attacha à conseiller les commerçants de cette rue sur divers changements souhaitables: les horaires de travail, la présentation de leurs vitrines, la promotion de leur produit, la qualité architecturale et esthétique et la valeur patrimoniale de leur édifice, le design possible pour retrouver,

"Mme Moulin est protesseure agregee au Departement des Sciences du Losir, al runiversité dontawa. généralement sous de multiples couches de pseudo-modernisme, les traits caractéristiques originels des bătiments, etc.

$\mathrm{Ce}$ premier projet connut une telle réussite qu'en 1984 le ministère de l'Expansion industrielle régionale du gouvernement fêdếral accorda une aide financière pour poursuivre et accroitre le programme en lui donnant une envergure pan-canadienne. Entre 1986 et 1991 , soixante-dix (70) municipalités canadiennes recevront de l'aide, par la Fondation, pour la revitalisation de leur centre-ville. Toutes les provinces, à l'exception de l'Ile du Prince-Edouard, participent à ce programme.

Entre 1979 et 1984 , sept projets furent développés dans des villes de l'Ontario, de l'Alberta et de la Colombie-Britannique. Au Québec, après avoir formé un groupe de personnes-ressources francophones, la revitalisation du centre-ville a pu s'effectuer dans deux municipalités, Ste-Marie de Beauce et St-Jean-sur-Richelieu. L'équipe québécoise a maintenant 16 projets à sa charge dans la province.

Dans chaque région du Canada, la rue principale possède ses propres caractéristiques ou particularites, ce qui lui donne son caractère et du caractère. Les variations sont dues en grande partie au mode de création et de développement de la ville, au type d'économie, aux valeurs sociales dominantes, à l'environnement physique et à son utilisation par les habitants. Les édifices construits le long de la rue principale ont conservé leurs messages culturels mais il reste à les déchiffrer et à les interpréter "),

Le programme Rues Principales comporte quatre volets pour assurer la revitalisation d'un centre-ville: l'organisation, la promotion, l'intervention, la relance.

Initialement, les marchands des centres-villes ne savaient pas comment coopérer en vue d'un objectif commun, ignoraient les règles du marketing et de la promotion. Le programme Rues Principales s'est attaché, depuis les premiers projets, à l'organisation d'une action des marchands; cette organisation, jointe à la promotion par le développement d'une image et d'une animation, permet lc développement commercial et économique du centre-ville.

Les projets furent d'abord confies à des architectes. Par leurs interventions sur les bâtiments et les espaces, ils permirent une grande amélioration esthétique. Dans tous les cas, cette intervention sur le băti, alliée à la promotion et à un marketing efficace, s'est avếrée très positive.

Depuis 1981, la Fondation s'applique à une formation de haute qualité pour ses chargés de projet. Elle publie un matériel didactique d'une durée de plusieurs semaines. Formés en architecture d'abord, les chargés de projets ont dû élargir leurs compétences pour s'adapter aux exigences de leurs tăches: celles-ci requièrent une combinaison savante de talents allant du design à l'animation, sans oublier le marketing, la conception des nouveaux produits et la promotion d'un programme varié d'activités.

\section{De la revitalisation au tourisme}

Une fois revitalisés, les centres-villes sont devenus des attractions touristiques importantes. Ce mouvement vers le tourisme n'était pas inscrit dans les objectifs initiaux du programme des Rues Principales. A Perth, en Ontario, la revitalisation a eu comme premier résultat le retour des résidents locaux sur leur rue principale; une reprise économique s'ensuivit qui encouragea les investissements en publicité. Ceci, en fin de compte, amena les touristes sur la rue principale. A Nelson, en ColombieBritannique, l'évolution a suivi le même cheminement.

La revitalisation, pour qu'elle soit réussie, doit se fonder sur la recherche de l'authenticité du patrimoine local - tangible et intangible. Cette authenticité doit être reconnue d'abord par les premiers concernés: les gens de la communauté. Les chargés de projets mettent en place, pour cela, des moyens de formation et d'animation. Ainsi, l'action des marchands ne peut plus être isolée de celle de la collectivité locale et se réduire à des opérations de cosmétiques seulement. S"ils veulent stimuler l'intérét pour leurs échoppes, ils doivent aussi épouser des intérêts qui sont finalement ceux du patrimoine. La communauté, en refaisant sa rue principale. renoue avec son passé, retrouve une fierté. Dans certains cas, comme à Nelson, la fierté s"accompagne d'une participation de la population aux réalisations de revitalisation. Par exemple, apres un cours auquel 85 personnes ont assisté, certaines d'entre elles ont connu le design de banes publics, de pierres taillées dans le respect du style propre à cette municipalité. L'acquisition de connaissances, de compétences, de techniques, d'aptitudes appliquées à la revalorisation de son patri- 
moine n'est certes pas un aspect négligeable du programme Rues Principales.

Dans ces conditions, que ce programme rejoigne les grands objectifs du tourisme culturel n'est pas finalement si surprenant.

\section{Culture et tourisme}

Faut-il rappeler le rơle déterminant de la culture dans toutes nos actions? L'Unesco n'a de cesse de ré-affirmer ce rôle dans l'ensemble des opérations de développement économique et social.

L'Association internationale des experts scientifiques du tourisine (AIEST) suggete que l'on recherche le développement de formes uniques et novatrices du tourisme et estime que dans tous les cas de développement touristique, les seuils de fréquentation, les capacités de charge, et l'intégration des équipements dans le milieu d'accueil doivent être respectés afin de maintenir une qualité sociale dans les rapports entre les visiteurs et leurs hôtes.

L'AIEST recommande également que les autorités touristiques régionales présentent toujours l'image authentique de leur destination pour parvenir à des stratégies de marketing plus efficaces.

Nous faisons face à deux aspects du tourisme.

Le véritable développement touristique devrait offrir à travers une connaissance approfondie du phénomène une possibilité de mise en valeur des ressources intellectuelles, affectives, spirituelles, physiques, rarement exploitées.

Lentement - peut-etre trop lentement - se dégage l'idée que le tourisme doit devenir educatif, se faire instrument de développement culturel et social, instrument d'information et de formation.

Il devrait stimuler l'individu et développer son inspiration, son imagination et sa créativité qui lui sont absolument nécessaires pour affronter l"avenir.

Un tourisme plus "culturel" serait donc une activité plus gratifiante pour le visiteur et pour le visité: il se fonderait sur la grande variété des cultures qui est une richesse et viserait à conserver les différences sociales. culturelles, ethniques, au lieu de tout standardiser.

La planification classique avait tendance a se faire pour une communauté et non avec elle. A l'heure actuelle, la communauté identifie ses points forts et définit des stratégies dans une demarche interdisciplinaire et prospective.

La vision du devenir de la communauté reste importante. Les ressources sont là. Le patrimoine construit d'un environnement, d'une cité, parle aux citoyens de cette communauté, leut raconte l"histoire, mais il est nécessaire qu'ils puissent la "lire" eux-mêmes avant d'en permettre la lecture aux autres.
Le patrimoine ne doit pas ètre considéré comme un bonus, une addition, un apport au produit touristique mais plutôt comme l'essence meme du produit touristique. Le patrimoine véhicule et représente; il est l'identité du pays, de la région. Il représente la façon dont ces individus ont géré leur espace et leur temps et ont su "inventer" leur société. En fait, le patrimoine est tout simplement l'aboutissement d'un projet, celui de nos ancêttres.

La relation entre tourisme et patrimoine 8 'avere rentable mais il faut comprendre que les deux sont intimement liés et intégrés au point que le tourisme ne pourrait pas se développer ni survivre sans cette prémisse de base qu'est le patrimoine culturel.

Le developpement du tourisme culturel associé à une communauté est alors signe de maturite. Il est nourri par les valeurs de la culture locale et se fait nourrissant par le processus qu'il déclenche et les possibilités créatrices d'initiatiwes qu'il promet.

Ce processus, puisqu'il fait appel à des activités de recherche, de formation et d'information favorise la maîtrise des savoir-faire et des savoir-être, l'interaction positive entre le savoir-faire traditionnel et l'environnement technique moderne; il est donc force d'integration et aussi vision d'avenir. Ainsi, il est force authentique et inaliénable.

La communauté ne devra donc plus préserver son identité, son style de vie, ses priorités face au développement touristique mais avec et par lui. En suivant les parametres culturels reconnus et établis comme prémisses au développement, les résidents décideront aisément du type et de l'échelle souhaitable du tourisme dans leur communauté.

Le tourisme culturel s'inserit dans la préservation et le renouveau des valeurs culturelles s'il est le résultat du processus nourrissant et enrichissant que nous avons décrit, car il redonne l'equilibre souhaite, il est construit sur les forces endogènes, senties et définies par la communauté elle-méme qui aura fait appell à toutes ses capacités créatrices, innovatrices.

Modernisation, revitalisation n'auront de sens que si I'on recherche cet équilibre dynamique, cette homéostasie entre les facteurs de changement et les nécessités d'assurer une continuité, donc préserver et renouveler les valeurs spirituelles, sociales, humaines et faciliter la communication interculturelle. Cette approche tend à considérer le touriste comme un individu que l'on accueillera dans le véritable accueil, 1'hospitalité, "l'hospes" retrouve(2)

Le développement touristique effectif d'une municipalité dépendra de ses ressources naturelles, culturelles et humaines. Il dépendra aussi de la taille de la région, des caractéristiques géographiques, ethniques, économiques et de son niveau de développement. Mais par dessus tout c'est le degré de connaissance de soi, les aptitudes à s'organiser, à créer et à avoir une vision de l'avenir qui sont importantes. Connaître d'abord et imaginer afin de répondre aux attentes des visiteurs, des touristes et leur fournir les éléments afin que soit dégagé un véritable sens du lieu et un esprit de ce même lieu. Élaborer un projet de sa communauté en devenir. Cultiver à travers le tourisme, son étude et son développement, toute la ressource humaine.

\section{Conclusion}

L intégration du développement touristique et de la revitalisation des centres-villes est concevable: les deux interventions poursuivent un même objectif: se reconnailtre pour mieux se definir, trouver sa place et faire des choix pour l'avenir. La dimension touristique dans ce cas consiste à inviter d'autres à partager nos atouts, à appréciér nos caractéristiques, à offrir des experiences pour que les comparaisons deviennent possibles. L'intégration du tourisme culturel à la revitalisation est véritablement un projet d"avenir".

Mais cette intégration exigera aussi que les intervenants de la revitalisation acquièrent la connaissance du phenomène touristique et de ses impacts sur la commmunauté. Concevoir des produits touristiques, assurer des hebergements convenables, poroposer des activités de temps libres, en tenant toujours compte de la variété des clientèles, de leurs besoins et de leur's désirs, tout cela ne s'improvise pas.

Le modele "Rues Principales" est en woie de devenir une approche pour le développement du tourisme culturel. Malgré l'expérience déảa acquise, une question reste en suspend: à quoi faut-il donner la priorité: au tourisme ou à la revitalisation? Initialement, comme on l'a vu à Perth ou à Nelson, les projets visaient à sauver le patrimoine pour ré-activer des commerces. Le processus qui fut ainsi déclenchế a rejoint celui du développement du tourisme culturel. Aujourd'hui, on pourrait dire que les Rues Principales sont d'abord des interventions en faveur du tourisme culturel. La Fondation d'ailleurs partage ces vues puisqu'elle élabore des stratégies de devveloppement touristique. Avec Rues Principales, elle a amorce un éveil irréversible dans les communautés locales et proposé à de nombreux individus de nouvelles approches et possibilites d'action. Stimulées par des occasions nouvelles de relance commerciale, plusieurs communautes et petites villes canadiennes se trouvent aujourd'hui engagés dans un programme de changement qui va beaucoup plus loin et qui peut desservir avantageusement le tourisme culturel $f$

\section{Notes explicatives:}

(1) KALMAN, $H_{1}$, Canada's Main Streets, 3-29, Reviving Main Street, Deryck Holdsworth Ed., Unwersity of Toronto Press, 1905.

(2) MOLLIN, Claude, Towistī Leusure, An Element of Mixing and Cutural Devolownent or Hospes Rediscovered, Revue Tourisme, no 2, 1985, 2-5. 